\title{
Dynamics of the Oort Cloud In the Gaia Era I: Close Encounters
}

\author{
S. Torres, S. Portegies Zwart and A. G. A. Brown \\ Leiden Observatory, Leiden University, PO Box 9513 Leiden, NL.2300 RA, the Netherlands. \\ email: storres@strw.leidenuniv.nl, spz@strw.leidenuniv.nl \& \\ brown@strw.leidenuniv.nl
}

\begin{abstract}
Comets in the Oort cloud evolve under the influence of internal and external perturbations from giant planets to stellar passages, the Galactic tides, and the interstellar medium.Using the positions, parallaxes and proper motions from TGAS in Gaia DR1 and combining them with the radial velocities from the RAVE-DR5, Geneva-Copenhagen and Pulkovo catalogues, we calculated the closest encounters the Sun has had with other stars in the recent past and will have in the near future. We find that the stars with high proper motions near to the present time are missing in the Gaia-TGAS, and those to tend to be the closest ones. The quality of the data allows putting better constraints on the encounter parameters, compared to previous surveys.
\end{abstract}

Keywords. Oort cloud, Solar System

\section{Introduction}

The outer regions of the Solar System are populated by a large number of planetesimals. Further away, more than 1,000 times farther than the Sun, and almost extending to the nearest stars, is the Oort cloud. It was proposed in the late 1950s by the Dutch astronomer Jan Hendrik Oort at the Sterrewacht Leiden, by anticipating its existence when he realised that long term comets $(a>40 \mathrm{AU})$ bound to the Sun must come from an area well beyond Neptune. Oort (1950) pointed out that a spike in the distribution of $1 / a$ of the long period comets with $a<10^{4}$ AU (see for example Wiegert \& Tremaine 1999), and isotropic distributions in $\cos i, \omega$, and $\Omega$, would argue for the existence of a reservoir of objects in quasi-spherical symmetry surrounding the Solar System. The external perturbations such as galactic tides, stellar flybys and molecular clouds play an important role in the formation and evolution of the Oort cloud. In particular, the role of passing stars is to reshuffle the comet distribution in the Oort could and to refill the high inclination region where comets are pushed into the planetary region. The GaiaDR1 (Gaia Collaboration et al. 2016) has opened a new window for the understanding of the Milky Way. In the particular case of the Solar System, Gaia detected the local star systems within 50pc from the Sun (compared to the $20 \%$ detected by Hipparcos (Perryman et al. 1997)). This allows for the stellar positions to be traced back far in time, which in turn allows reconstructing the history of stellar encounters and its implication for the comets of the Oort cloud.

\section{Method}

Using the positions, parallaxes and proper motions from TGAS (Gaia Collaboration et al. 2016) and combining them with the radial velocities from the RAVE-DR5 (Kunder et al. 2017), Geneva-Copenhagen (Nordström et al. 2004), and Pulkovo (Gontcharov 2006) catalogues, we computed the $6 \mathrm{D}$ elements for the stars in the immediate solar 

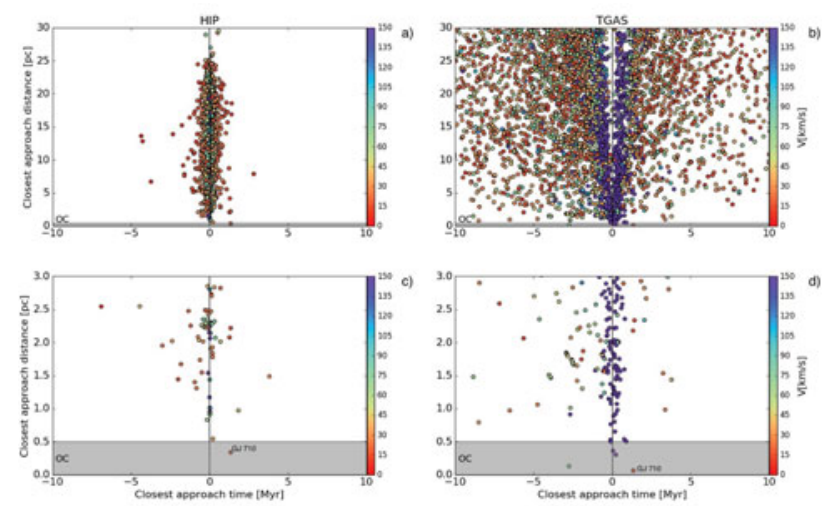

Figure 1. Closest approach of distance vs time for stars within 30pc (a -Hipparcos data-, b -TGAS data-) and 3 pc (c-Hipparcos data-, $\mathbf{d}$-TGAS data-) with respect to the Sun; colorbar represents the velocity of the stellar approaches.

neighbourhood $(<30 \mathrm{pc})$ backwards and forward in time $( \pm 10 \mathrm{Myr})$ to identify the past and future solar encounters. The orbital integration of the stars was performed by using an axisymmetric potential included in the module Galaxia in AMUSE (Pelupessy et al. 2013).

\section{Results/Discussion}

The closest encounter to the Sun has been studied for years by taken advantage of the Hipparcos catalogue (see for example García-Sánchez et al. 2001). It has been a general agreement of the potential perturbers to the Sun, particularly with the star HIP 89825 (Gliese 710) that according to García-Sánchez et al. 2001 will perturb the Sun in 1.36 Myr to within $0.33 \mathrm{pc}$ respect to the Sun (Figure $1 \mathrm{a}, \mathrm{c}$ ); however the Hipparcos data is incomplete. Using TGAS, we recalculate the encounters history of the Sun and the stars in the solar neighbourhood (Figure $1 \mathrm{~b}, \mathrm{~d}$ ) and we find that Gliese 710 will approach the Sun in 1.35Myr to within 0.06pc (see also Berski and Dybczyński 2016). Due to the better completeness of the Gaia data, we increased the number of encounters within 30 pc, and we find a distribution of the closest stars with better accuracy. However, we are missing the recent encounters with high proper motions, which are missing from the TGAS catalogue.

This work has made use of data from the European Space Agency (ESA) mission Gaia (https://www.cosmos.esa.int/gaia).

\section{References}

Berski, F. \& Dybczyński, P. A. 2016, A\&A, 595, L10

García-Sánchez, J., Weissman, P. R., Preston, R. A., et al. 2001, A\&A, 379, 634

Gaia Collaboration, Brown, A. G. A., Vallenari, A., et al. 2016, A\&A, 595, A2

Gontcharov, G. A. 2006, Astronomy Letters, 32, 759

Kunder, A., Kordopatis, G., Steinmetz, M., et al. 2017, AJ, 153, 75

Nordström, B., Mayor, M., Andersen, J., et al. 2004, A\&A, 418, 989

Oort, J. H. 1950, bain, 11, 91

Pelupessy, F. I., van Elteren, A., de Vries, N., et al. 2013, A\&A, 557, A84

Perryman, M. A. C., Lindegren, L., Kovalevsky, J., et al. 1997, A\&A, 323, L49

Wiegert, P. \& Tremaine, S. 1999, Icarus, 137, 84 